\title{
Low testosterone increases risk of cardiovascular events in women
}

Low levels of testosterone are associated with increased all-cause mortality and cardiovascular events in a cohort of German women, according to a study by Caroline Sievers (Max Planck Institute of Psychiatry, Munich, Germany) and colleagues.

"Men have a higher risk of cardiovascular morbidity and mortality than women; therefore, raised testosterone levels could have adverse effects on the cardiovascular system in women," says Sievers. However, "the predictive value of testosterone on cardiovascular events and mortality in women has not yet been evaluated in large cohorts."

In a prospective cohort study, the researchers analyzed the main outcome measures (cardiovascular risk factors, cardiovascular disease and all-cause mortality) in 2,914 women over a 4.5 -year follow-up period.

The researchers found that those patients with the lowest levels of testosterone
$(<0.82 \mathrm{nmol} / \mathrm{l})$ had the highest risk of a cardiovascular event or all-cause mortality, independent of traditional risk factors. These results imply that "low testosterone is associated with adverse effects on women's cardiovascular risk profile," says Sievers.

The researchers suggest two hypotheses to explain their findings. Low testosterone might cause or worsen pre-existing disease, leading to increased mortality rates. Alternatively, low testosterone could simply be a marker of disease. The investigators suggest this study should be replicated in other populations and the underlying mechanisms explored in animal or in vitro models.

\section{Claire Greenhill}

Original article Sievers, C. et al. Low testosterone levels predict all cause mortality and cardiovascular events in women; a prospective cohort study in German primary care patients. Eur. J. Endocrinol. 163, 699-708 (2010) 